\title{
CONSTRUCCIÓN DEL CONCEPTO DE LÍMITE: UN ESTUDIO DE CASO*
}

Por:

Rosa Elvira Páez Murillo**

\section{RESUMEN}

El objetivo de este estudio fue investigar la construcción del concepto de límite en estudiantes de posgrado en un ambiente de aprendizaje cooperativo, de debate científico y de autorreflexión. Se diseñaron veintidós actividades, las cuales se desarrollaron durante catorce sesiones de dos horas y media cada una. La dinámica consistió en impulsar la participación de los estudiantes organizándolos en grupos pequeños para que resolvieran las tareas incluidas en las actividades. Después, todos los miembros del grupo participaban en un debate científico para revisar y analizar los enfoques diversos de los equipos. Se pedía a los estudiantes que fuera de clase llevaran a cabo una reflexión de las tareas desarrolladas en el salón. Al finalizar todas las sesiones de trabajo, resolvieron individualmente un examen y una semana después se realizó una entrevista con cada uno de ellos. En este artículo se presenta aspectos generales y particulares de la investigación y el análisis de un estudio de caso.

\section{ABSTRACT}

The aim of this study was to investigate students' reconstruction of the limit concept in a scientific debate/ cooperative learning environment. We designed twentytwo activities, which were developed during fourteen sessions of two hours each one. The dynamics consisted of first stimulating the active participation of the students in a discussion in small groups in order to complete the tasks included in the designed activities. In the second term, all the members of the group were taking part in a scientific debate in order to check and to analyze the approaches of each team. In the third and final part, the students were asked to think about the tasks developed in the classroom. In this article we will present general aspects of the investigation and a case study.

Palabra claves: Límite, Metodología de enseñanza, Aprendizaje cooperativo.

\section{INTRODUCCIÓN Y MARCO TEÓRICO}

La historia de las ideas matemáticas muestra que el concepto de límite es complejo. "La definición de límite en términos de $\varepsilon$ y $\delta$ el resultado de más de cien años de ensayo y error, e incorpora en unas pocas palabras el fruto de un esfuerzo persistente para dotar a este concepto de una base matemática sólida... Sin embargo, una comprensión clara y una definición precisa de los límites estuvieron bloqueadas durante largo tiempo por una dificultad aparentemente insuperable" (Courant y Robbins, 1941, p. 342 en la ed. en español). De la misma manera, la historia revela los obstáculos que tuvieron determinados matemáticos para entender y formalizar el concepto de límite (Cajori, 1915; Grattan-Guinness, 1970; Bell, 1940) de ahí, entonces que sea más que natural que se presenten también dificultades en su aprendizaje en los estudiantes de cualquier nivel. En este contexto, el interés se enfocó en investigar dificultades de aprendizaje y procesos de construcción o reconstrucción del concepto de límite en estudiantes de posgrado que han ejercido como profesores de matemáticas y/o de cálculo.

Investigadores en educación matemática, como Cornu (1981 y 1994), Sierpinska (1985, 1987 y 1988), Tall y Schwarzenberger (1978), Hitt y Páez (2001, 2003,

\footnotetext{
* Los resultados descritos en este reporte de investigación son parte de uno de los diecisiete casos analizados en la tesis doctoral dirigida por el Dr. Fernando Hitt (Cinvestar - Universidad de Québec)

** Universidad Francisco de Paula Santander, Cúcuta-Colombia. Centro de Investigación y de Estudios Avanzados del Instituto Politécnico Nacional. (Cinvestar) rpaez@cinvestav.mx
} 
Construción del concepto de límite: Un estudio de caso

2004), entre otros, se han preocupado por identificar la problemática relativa a las dificultades que tienen los estudiantes en la construcción del concepto de límite. Cornu y Sierpinska han relacionado estas dificultades con aspectos históricos del concepto de límite, estableciendo así obstáculos de corte epistemológico.

La investigación se enmarcó desde una perspectiva de la construcción de conceptos, fundamentada en la teoría de representaciones semióticas (Duval, 1998 y 1999; Hitt, 2003). En particular, se consideró de suma importancia las producciones semióticas de los estudiantes en el proceso de construcción del concepto de límite. Otro aspecto esencial fue la posibilidad de detectar en ellos procedimientos que reflejen cierto tipo de conocimiento, al que se identificó como una concepción' en el sentido de Duroux (1983). Este conocimiento funciona localmente y dependiendo de la situación el estudiante puede resolver un problema y en otras situaciones, ese conocimiento, promueve el error.

Para fines de la investigación, se estableció como concepción el conocimiento del alumno con respecto a un concepto, útil en determinadas situaciones matemáticas y en otras lo lleva al fracaso. Este conocimiento funciona en el estudiante como una unidad, cada vez que se enfrenta a un problema, y se manifiesta a través de sus producciones semióticas. Algunas concepciones provocarán un obstáculo epistemológico en el sentido de Brousseau (1983) y Sierpinska (1985). Un ejemplo de una concepción que se vuelve obstáculo es el siguiente: en un estudiante de primer semestre de universidad (Páez, 2001) identificamos que tiene la concepción de límite como sustitución, cuando en un ejercicio de la forma $\lim _{x \rightarrow 3} \sqrt{x+1}$ responde que el límite es dos porque sustituye el valor de a en la expresión. Esta concepción le permite encontrar la respuesta, cuando las funciones son continuas. Pero, en el caso contrario lo conduce a un error, como en el ejemplo utilizado de una representación gráfica de una función discontinua, donde prevalece la sustitución y si el punto de discontinuidad es a, el estudiante afirma que el límite cuando $x$ tiende a a es $f(a)$.

Las preguntas de investigación que orientaron este estudio fueron: ¿̇ué concepciones de límite presentan algunos estudiantes de posgrado? y ¿Qué avances con respecto al proceso de construcción o de reconstrucción del concepto de límite se pueden observar en los estudiantes de posgrado frente a situaciones no rutinarias en un ambiente de aprendizaje cooperativo, de debate científico y de autorreflexión?

\section{METODOLOGÍA}

Los sujetos que participaron en el estudio fueron diecisiete estudiantes de una maestría de educación matemática que han ejercido como profesores de matemáticas y/o de cálculo.

Para lograr el objetivo propuesto en esta investigación, se diseñaron veintidós actividades, las cuales incluían diferentes representaciones del concepto de límite (gráfica, numérica, algebraica y en lenguaje natural). Para su resolución necesariamente se verían obligados a realizar procesos de conversión entre representaciones. Por ejemplo, en una de las actividades la cual está referenciada en el trabajo de investigación como la No. 7, el estudiante tiene que integrar dos representaciones, la geométrica y el lenguaje natural, para comprender el problema. Luego, para su solución es indispensable un cambio a la representación algebraica.

1 Duroux (1983) define este término como un saber local que se produce cuando se privilegian ciertas situaciones, en menoscabo de otras, en la adquisición del conocimiento. Por lo tanto, una concepción es operante sobre una parte de lo que Vergnaud designa como campo conceptual y por ende presenta necesariamente insuficiencias. 


\section{Actividad No. 7}

Partiendo de un cuadrado grande, trace otro al interior desplazando sus vértices como se indica en la figura: cada vértice está sobre un lado del primer cuadrado a una distancia igual a $1 \mathrm{~cm}$

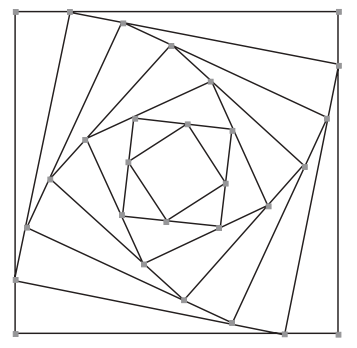
de su vértice. Dibuje otro cuadrado siguiendo el mismo proceso y después otro más y así sucesivamente.

a. ¿̇Hasta dónde se puede realizar esta construcción?

b. Compare este problema con el de los cuadrados construidos en los puntos medios de los lados de cada uno de los cuadrados.

c. Compare este problema con el de los hexágonos y dodecágonos.

La actividad fue diseñada para discernir dos posiciones, para unos es un proceso finito y para otros se trata de un proceso infinito ligado al infinito potencial (Páez, 2004). La controversia se podrá resolver cuando pasen al terreno algebraico. De hecho, el paso a la representación algebraica se presenta como necesario para poder argumentar a favor o en contra, ya que en el ámbito perceptivo, la discusión no podría ir muy lejos. Es importante señalar, que con este problema, la propiedad de iniciar con un cuadrado e ir construyendo otro cada vez, el límite es también un cuadrado. En otros problemas se había llegado a la conclusión que el límite era un punto (actividades con cuadrados y hexágonos).

Las actividades se desarrollaron durante catorce sesiones de dos horas y media cada una. La metodología utilizada en el desarrollo del curso fue la del debate científico (Alibert y Thomas, 1994; Legrand, 2001) en un ambiente de aprendizaje cooperativo (Hagelgans, et. al, 1995) y de reflexión personal.
Para seguir las indicaciones de la metodología del aprendizaje cooperativo, con respecto a la conformación de los grupos y con relación al nivel académico, se aplicó un cuestionario diagnóstico. De acuerdo a los resultados, se clasificó a los estudiantes de posgrado según su desempeño, en tres tipos:

1. Los estudiantes que demostraban afirmaciones no contradictorias en sus respuestas y buen desempeño en general.

2. Los estudiantes que planteaban afirmaciones contradictorias en sus respuestas.

3. Los estudiantes que mostraban limitaciones en su conocimiento pero sin contradicciones en sus repuestas.

Una vez realizada la clasificación se procedió a formar los equipos de trabajo, incluyendo un miembro de cada uno de los tres grupos y procurando que en cada uno hubiese estudiantes cuya formación inicial fuese de matemática o de ingeniería.

En cada sesión, había un tiempo para que los miembros de los pequeños grupos discutieran y desarrollaran la o las actividades planteadas. Éstas fueron diseñadas para fomentar la participación dentro de los equipos. En ellos, cada uno asumiría un rol diferente por sesión. Por ejemplo, uno manejaría la calculadora; otro, asumiría el papel de redactor y un tercero el de expositor. La finalidad fue permitir la interacción entre ellos.

Cuando cada equipo llegaba a "un consenso", se pasaba a la discusión general aplicando un punto de vista particular de la llamada metodología del debate científico, en el sentido de Alibert y Thomas (1994) y Legrand (2001). La discusión general ayudaba a reafirmar o a cambiar la posición que tenía cada uno dentro de sus pequeños grupos. Al final de cada sesión, los equipos debían entregar todos los ensayos realizados en la búsqueda de la solución del problema y a la siguiente clase entregar un informe individual (fase importante de reconstrucción individual de lo realizado en el pequeño grupo y en el debate). En la última sesión los estudiantes presentaron un examen escrito y una semana después se les hizo la entrevista. 
Construción del concepto de límite: Un estudio de caso

En resumen, los pasos importantes que se siguió con esta metodología fueron los siguientes:

- Los estudiantes trabajaban sobre las tareas en pequeños grupos, en un ambiente de aprendizaje cooperativo.

- Los alumnos participaban en una discusión plenaria con la totalidad de la clase en un ambiente de debate científico. Luego, entregaban todo lo que habían realizado en la sesión (las diferentes propuestas, dibujos, etc.).

- Los estudiantes reexaminan la actividad (en una reflexión personal de reconstrucción como tarea) basado sobre sus trabajos previos (discusión en los pequeños grupos y con la totalidad de la clase).

- Los alumnos presentaron un examen final y participaron en una entrevista.

\section{RESULTADOS}

A continuación se presenta un caso: el de "Pedro", cuya formación inicial es de licenciado en matemáticas y física. Se muestra brevemente el trabajo realizado por este estudiante, especialmente en el examen y en la entrevista.

a. Cuestionario diagnóstico y sesiones de clase: Pedro inició con deficiencias en el concepto de función, por ejemplo, dificultades para identificar el dominio y el rango, graficar funciones racionales y realizar cambios de registro. Aunque estaba inmerso en un ambiente de aprendizaje cooperativo y debate científico, a lo largo de las sesiones fueron evidentes las concepciones y dificultades que presentó. Por ejemplo, la concepción de que para cada épsilon basta tomar el delta igual a épsilon. Concepción que mantuvo a pesar de los ejemplos, explicaciones del profesor y del trabajo en pequeños grupos. Esta estrategia funciona para algunos casos (por ejemplo, $f(x)=x+c$ siendo $c$ una constante). La dificultad estriba en que él pretendió aplicar esta estrategia en todos los casos. Es importante señalar que en su desempeño prevaleció una tendencia a participar poco con respecto al trabajo en grupo, él no estaba acostumbrado a una metodología como la utilizada y no se integró plenamente a su pequeño grupo de discusión; sin embargo, participaba en el debate regularmente.

b. Examen: En una de las preguntas del examen se solicitó que proporcionara una definición de límite. Pedro suministró las siguientes (ver Figura 1):

$$
\begin{aligned}
& \text { Un valor real of wen fancion } f(x) \text { as } \\
& \text { igual a L esto quiere becir } \\
& \text { que } \forall \varepsilon>0 \quad J \delta>0 \text { tal que } 0<|x-a|<\delta \\
& \text { siompre gue }|f(x)-L|<\varepsilon \text { esto } \\
& \text { ba) es paror limite de unn funciré } \\
& \text { purn limite de unn Sucvión } \\
& \forall \varepsilon>0 \quad \exists N \in \mathbb{N} \text { con } n>N \text { talque }\left|a_{m}-L\right|<\varepsilon \\
& \text { siempre que } n>N \text {. }
\end{aligned}
$$

Figura 1

Como se puede observar, en la definición de límite de una función, cambió el orden de la implicación, es decir, afirmó que $|f(x)-L|<\varepsilon \Rightarrow|x-a|<\delta$. Este fue un error muy común de los estudiantes que proporcionaron una definición incorrecta. El error puede provenir de la forma en cómo se presenta la definición. Por ello se revisó algunos libros de cálculo y se identificó cómo expresan la definición de límite de una función en un punto. En el cálculo de Leithold (1982), Spiegel (1978), Kitchen (1986), Larson y Hostetler (1986), y Stewart (1999), la muestran así: "... $|f(x)-L|<\varepsilon$ siempreque $0<|x-a|<\delta$ ", la cual podría generar justamente esa dificultad de cambiar el orden de la implicación, de ahí, que algunos estudiantes piensen que "... $|f(x)-L|<\varepsilon \Rightarrow|x-a|<\delta$ ". Se piensa que la mejor manera, desde un punto de vista educativo, de definir el límite de una función en un punto, es como aparece en los libros de cálculo de Spivak (1999), Stein (1982), Swokowski (1982), y como se presentó en el curso, es decir, de la forma si $p \Rightarrow q$ ("...si entonces $0<|x-a|<\delta$ entonces $|f(x)-\boldsymbol{L}|<\varepsilon^{\prime \prime}$ ). 
Construción del concepto de límite: Un estudio de caso

Retomando el análisis de la definición que proporcionó Pedro en el examen, se puede conjeturar que se aprendió una definición de memoria y al quererla recordar, recupera sólo algunas partes de ésta. Es evidente que no construyó una articulación entre la definición y otro tipo de representaciones que le pueda ayudar a no olvidar la definición.

Es interesante el hecho que después de cometer este error, proporciona la definición del límite de una sucesión colocando correctamente el orden de la implicación. Puede ser que se deba a que en el curso se enfatizó más en sucesiones o se aprendió bien la definición.

En la segunda parte del examen se le pidió trazar una gráfica de la función $f$ que cumpliera las siguientes condiciones:

\begin{tabular}{|ll|lc|}
\hline a. & $\lim _{x \rightarrow 2^{-}} f(x)=0$ & b. & $\lim _{x \rightarrow 0^{+}} f(x)=-1$ \\
\hline c. $\quad \lim _{x \rightarrow 0^{-}} f(x)=1$ & d. & $\lim _{x \rightarrow 2^{+}} f(x)=1$ \\
\hline e. & $f(2)=1$ & f. & $f(0)=$ no está definido \\
\hline
\end{tabular}

Pedro dibujó una gráfica que cumplía sólo con las condiciones a), b) y e). $O$ más bien, marcó puntos y los unió con una línea recta (ver Figura 2). El que no haya podido construir la función solicitada es indicio que tiene dificultades para pasar del registro algebraico al gráfico.

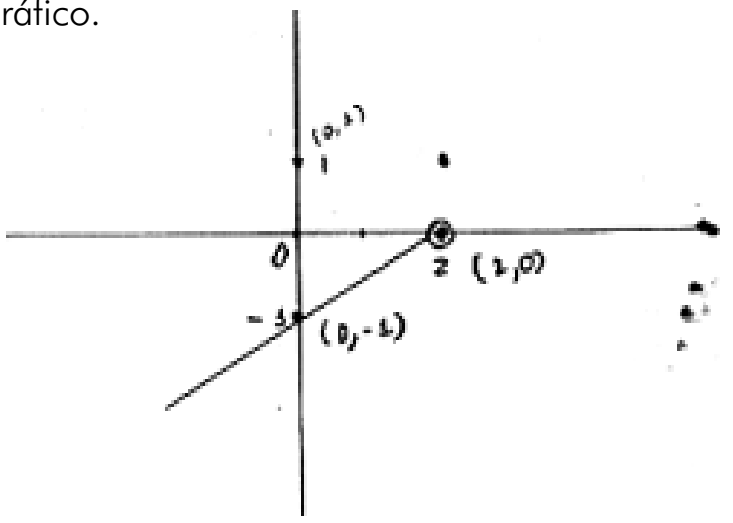

En la representación gráfica, el marcar con puntos el $\lim _{x \rightarrow 2^{-}} f(x)=0$ y $\lim _{x \rightarrow 0^{+}} f(x)=-1$, es una señal para considerar que Pedro posee la concepción de límite como una simple sustitución. Es decir, considera el límite como el valor que toma la función, en el punto que se está analizando, sin importar si la función es continua o no (el límite es el valor que se obtiene al sustituir $a$ en la función $\left.\mathrm{f}(\mathrm{x}), \lim _{x \rightarrow a} f(x)=f(a)\right)$. En ello, no hay un análisis del comportamiento de la función en vecindades que contengan al punto. Es el reflejo de considerar el proceso al infinito como una simple sustitución. Concepción que conduce a una respuesta correcta, cuando la función es continua, pero en el caso contrario lleva a una respuesta errónea.

Esta conjetura sobre su concepción se corroboró con uno de los ejercicios que se propuso en el examen. Se le preguntó por el $\lim _{x \rightarrow a} f(x)$ donde $f(x)=\left\{\begin{array}{ll}1 & \text { si } x \text { es entero } \\ 0 & \text { si } x \text { no es entero }\end{array}\right.$ Él escribió que por unicidad del límite no existe. Consideró que hay un límite para los $x$ enteros y uno para los $x$ no enteros. Supone la existencia de dos límites, porque sustituye el valor $a$ en cada una de las expresiones que componen la función. Además, hace una mala interpretación del teorema: "Una función no puede tender hacia dos límites diferentes en $a^{\prime \prime}$ (Spivak, 1999, p. 120).

Del mismo modo se hizo evidente que Pedro interpretó mal el siguiente teorema: "Suponga que la función $f$ está definida en una vecindad perforada del punto $a$. Entonces el $\lim _{x \rightarrow a} f(x)$ existe y es igual al número $L$ sí y sólo si los límites laterales $\lim _{x \rightarrow a^{-}} f(x)$ y $\lim _{x \rightarrow a^{+}} f(x)$ existen y son iguales a $L$ (Edwards y Penney, 1996, p.75)". Pedro no se percata que $L$ debe ser un número real, afirma que sí existe el $\lim _{x \rightarrow c} f(x)$ cuando $\lim _{x \rightarrow c^{+}} f(x)=+\infty=\lim _{x \rightarrow c^{-}} f(x)$ 
Construción del concepto de límite: Un estudio de caso

c. Entrevista: La entrevista fue de tipo semiestructurada. Allí se le solicitó que proporcionara un ejemplo de una sucesión convergente y una divergente. Él inició con una sucesión divergente y suministró las definiciones de convergencia y no-convergencia (ver Figura 3). En la definición de convergencia olvidó hacer

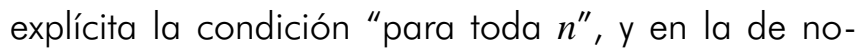
convergencia omitió el "existen algunos $n$ ". Luego pasó a realizar la representación gráfica, para explicar, a través de ella y de la idea intuitiva de vecindad, que la sucesión diverge. Como se puede observar en la Figura 3, Pedro representó la gráfica de una sucesión como continua y no discreta, como debería ser.

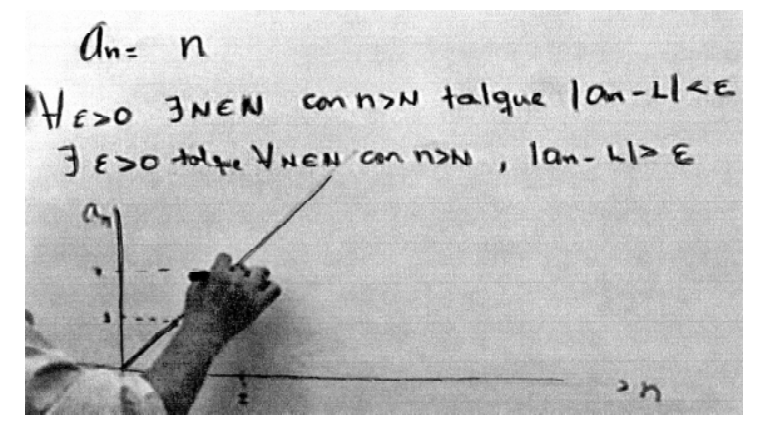

Figura 3

Sus dificultades mayores iniciaron cuando entró a lo formal. Empezó la demostración de que la sucesión $n$ diverge sin especificar las condiciones del contradominio y del dominio (ver Figura 4) y tuvo dificultades con el valor absoluto. Se advierte que él opera algebraicamente sin un referente geométrico, puesto que olvidó por completo la gráfica que lo ayudó a explicar su idea intuitiva.

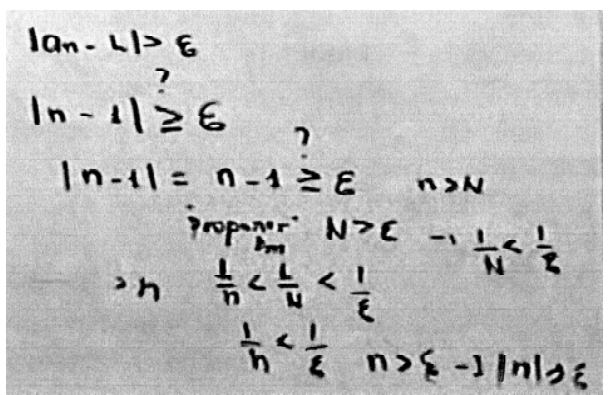

Figura 4

Después de hacer el desarrollo algebraico que se ilustra en la Figura 4 se presentó el siguiente diálogo con el entrevistador:
Pedro (P): Ya con esto estoy demostrando que no puedo encontrar un valor que pueda encerrarlo en el límite que he considerado. Y así sucesivamente. Si lo tomó para 2 , para 3 , no voy a encontrar, lograr meterlo dentro de esa vecindad, dentro de ese entorno.

Entrevistador (E): Pero no tiene que ver que tú tomaste como un ejemplo particular el uno, no tendría que ver que el $|n-1|$ es mayor o igual que $\varepsilon$, no que el $n$, porque dado cualquier $\boldsymbol{\varepsilon}$, siempre puedo encontrar un $n$ grandote.

P: Ah!!!!!! Sí, si, si... tienes toda la razón [Y realizó el siguiente proceso]

$$
\begin{aligned}
& \mathrm{n} \geq \varepsilon+1 \\
& \mathrm{n}>\mathrm{N} \\
& \mathrm{N} \geq \varepsilon+1 \\
& \frac{1}{N} \leq \frac{1}{\varepsilon+1} \\
& {[\text { como ] } \mathrm{n}>\mathrm{N}} \\
& \frac{1}{n}<\frac{1}{N} \leq \frac{1}{\varepsilon+1} \\
& \frac{1}{n} \leq \frac{1}{\varepsilon+1} \\
& n \geq \varepsilon+1 \\
& n-1 \geq \varepsilon
\end{aligned}
$$

Entonces si lo introduzco en valor absoluto quedaría que regreso a demostrar que $|n-1| \geq \varepsilon$

E: ¿̇qué pasa si tomas un $\varepsilon=500$ ?

P: $\varepsilon=500,|n-1| \geq 500$ Entonces el $N$, para que esto se cumpla... si $\varepsilon=500 \ldots$ ese $N$ tiene que ser mayor que $500 \ldots$

E: Pero en la negación dice que para toda $N$, żpor qué tenemos que tomar $N>500$ ? Si es para toda $N$...

En el momento en que el entrevistador le hace notar que está restringiendo el valor de $N$, Pedro entró en 
contradicciones, a tal punto que para salvaguardar lo que desarrolló, aceptó que cometió errores, al proporcionar las definiciones, y modificó el cuantificador $\exists N$ por $\forall N$ en la de convergencia y viceversa en la de divergencia (ver Figura 5)

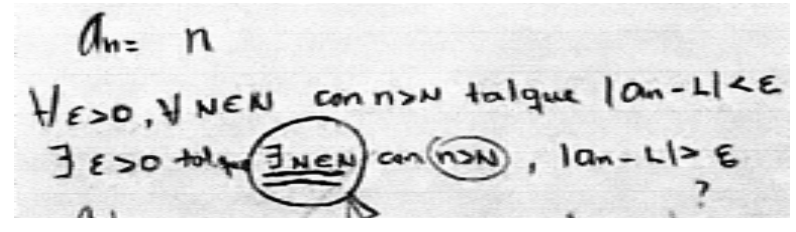

Figura 5

Pedro tiene una idea intuitiva que no fue capaz de formalizar. El problema es que él separa lo intuitivo y lo formal. Cuando entra a lo formal no se da oportunidad de que lo oriente su idea representada en la gráfica. Además, prefiere aprenderse las definiciones de memoria que utilizar la gráfica, para tener un referente geométrico, al momento de evocarlas.

Se retoma la pregunta sobre el $\lim _{x \rightarrow a} f(x)$ de la función $f(x)=\left\{\begin{array}{rr}1 & \text { si } x \text { es entero } \\ 0 & \text { si } x \text { no es entero }\end{array}\right.$

En el examen respondió que este límite no existe porque el teorema de la unicidad no permite que tenga dos límites diferentes. Aquí mencionó de nuevo lo mismo, es decir, él afirmó que cuando $a$ es entero el límite es uno y cuando $a$ no es entero es cero. Sólo que a esta dificultad se le puede añadir tres más, que surgieron en el desarrollo de la explicación. La primera fue que dibujó el $\varepsilon$ en el eje de las $x$ (ver Figura 6). La segunda fue cuando estaba analizando el límite por la derecha de 0.99, aquí manifestó que en esa vecindad se debía de considerar dos casos: un límite para los no enteros y el límite cuando se analiza estrictamente el uno. La última fue que $a$ va variando.

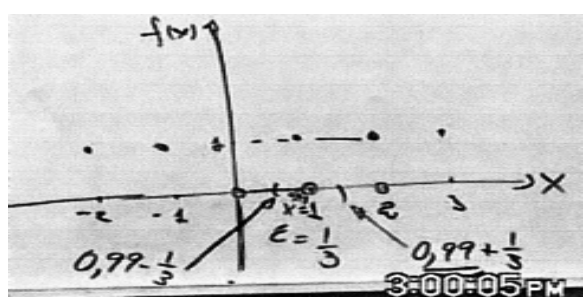

Figura 6

\section{DISCUSIÓN}

El análisis realizado permite afirmar que Pedro no tiene una construcción adecuada del concepto de límite y que la metodología, a corto plazo, no tuvo el impacto que se esperaba en él. Probablemente se deba a que es un estudiante acostumbrado a aprenderse los procesos de memoria, sin ningún significado geométrico. Pedro prefiere la metodología tradicional, en donde se hace énfasis a la representación algebraica. Pareciera que su buen desempeño en cursos anteriores es porque bajo una metodología tradicional, es costumbre aprenderse las definiciones de memoria y realizar algunos ejercicios rutinarios. Además, sus conceptos previos no están bien fundamentados, precisamente porque no hay una articulación entre las diferentes representaciones. La conceptualización implica una coordinación de registros de representación (Duval 1998), libre de contradicciones (Hitt, 2001).

Se ha querido mostrar un caso donde es claro que la metodología no funcionó del todo. Pedro estaba acostumbrado a aprender de memoria definiciones y demostraciones de teoremas, y las actividades en general eran de tipo no rutinario. Pero en términos generales se puede afirmar que la metodología utilizada en esta experimentación resultó positiva para la mayoría de los estudiantes (Páez, 2004).

\section{AGRADECIMIENTOS}

La autora agradece los comentarios y sugerencias a este trabajo de investigación realizados por el Dr. Fernando Hitt (México) y el Dr. Francois Pluvinage (Francia). Asimismo expresa sus agradecimientos al gobierno de México por la beca otorgada a través de la Secretaría de Relaciones Exteriores para realizar los estudios de doctorado, y a la Universidad Francisco de Paula Santander por conceder la comisión Ad Honorem. 
Construción del concepto de límite: Un estudio de caso

\section{REFERENCIA BIBLIOGRÁFICA}

Alibert, D. \& Thomas, M. (1994). Research on mathematical proof. In D. Tall (Ed.) Advanced Mathematical Thinking, pp. 215-230. Kluwer Academic Publishers.

Bell, E. (1940) The development of Mathematics. Traducción al español por Fondo de Cultura Económica, México, 2003.

Brousseau, G. (1983). Les obstacles epistemologiques et les problèmes en mathematiques. Recherches en Didactique des Mathematiques.Vol. 4 No. 2. pp. 165 198.

Cajori, F. (1915). The history of Zeno's Arguments on Motion: Phases in the Development of the Theory of Limits. American Mathematical Monthly, Vol. XXII, pp. 1-6, 39-47,77-82, 109-115, 143-149, 179-186, 215-220, 253-258, 292-297. Traducción al español por Elisa Zacarías. Revista del seminario enseñanza y titulación. Año IV. Octubre 1987.

Cornu, B. (1981). Apprentissage de la notion de limite: modèles spontanés et modèles propres. Proceedings PME-V, Grenoble, France, Vol. I, pp. 322-326.

Cornu B. (1994). Limits. In D. Tall (Ed.) Advanced Mathematical Thinking, pp. 153-167. Kluwer Academic Publishers.

Courant, R. y Robbins, H. (1941) What is Mathematics? Traducción al español por Fondo de Cultura Económica, México, 2002.

Duroux, A. (1983). La valeur absolve: Difficultés majeures pour une notion mineure. Petit $X$, No. 3, pp. 43-67.

Duval R. (1999) Sémiosis y pensamiento humano. Registros semióticos y aprendizajes intelectuales. Universidad del Valle.

Duval, R. (1998). Registros de representación semiótica y funcionamiento cognitivo del pensamiento.
Investigaciones en Matemática Educativa II. Grupo Editorial Iberoamérica, México, pp. 173-201.

Edwards, C. Y Penney, D. (1996). Cálculo con geometría analíica. Prentice Hall.

Guinness, G. (1970). The development of the foundations of mathematical analysis from Euler to Riemann. The MIT press.

Hagelgans, N., Reynolds, B., Schwingendorf, K., et al. (1995). A Practical Guide to Cooperative Learning in Collegiate Mathematics. MAA Notes Number 37.

Hitt, F. (2001). El papel de los Esquemas, las Conexiones y representaciones Internas y Externas Dentro de un Proyecto de Investigación en Educación Matemática. En Iniciación a la investigación en Didáctica de la Matemática. Homenaje al profesor Mauricio Castro (P. Gómez \& L. Rico Editores). Universidad de Granada, pp. 165-177.

Hitt, F. (2003). Le caractère fonctionnel des représentations. Annales de Didactique et de sciences cognitives, Vol. 8, pp. 975-999, IREM de STRASBOURG.

Hitt, F. y Páez, R. (2001). The notion of limit and learning problems. Proceedings PME-NA XXIII, Utah, USA, 2001, Vol. 1, pp. 169-176.

Hitt, F. y Páez, R. (2003). Dificultades de aprendizaje del concepto de límite de una función en un punto. Revista Uno, No. 32, pp. 97-108.

Hitt, F. y Páez, R. (2004). On the limit concept in a cooperative learning environment: A case study. Proceedings PME-NA XXVI, Toronto, Canadá, 2004, Vol. 1, pp. 103-110.

Kitchen, J. (1986). Cálculo. Mc Graw-Hill.

Larson, R. y Hostetler, R (1986). Cálculo y geometría analítica. Mc Graw-Hill 
Construción del concepto de límite: Un estudio de caso

Legrand, M (2001) Scientific debate in mathematics courses. Teaching and Learning of Mathematics at University Level. An ICMI Study. Edited by Derek Holton. pp. 127-135.

Leithold, L. (1982). El cálculo con geometría analítica. Cuarta edición.

Páez, R. (2001). Dificultades de aprendizaje en el concepto de límite: Ideas del infinito. Tesis de Maestría. Cinvestav-IPN. México.

Páez, R. (2004). Procesos de construcción del concepto de límite en un ambiente de aprendizaje cooperativo, debate científico y autorreflexión. Tesis de Doctorado. Cinvestav-IPN. México.

Sierpinska, A. (1985). Epistemological obstacles relative to the limit concept. Obstacles epistemologiques relatifs a la notion de limite. Recherches en didactique des mathématique. Vol. 6 No. 1, pp. 5-67.

Sierpinska, A. (1987). Humanities students and epistemological obstacles related to limits. Educational Studies in Mathematics, Vol. 18, pp. 371-397.

Sierpinska, A. (1988). Sur la relativité des erreurs. The role errors play in the learning and teaching of mathematics. En Compte rendu de la 39e rencontre internationale de la CIEAEM. Sherbrooke, Canada. pp. 70-87.

Spiegel, M. (1978). Teoría y problemas de cálculo superior. Serie de compendios Schaum. Mc Graw-Hill.

Spivak, M. (1999). Cálculo infinitesimal. Segunda edición. Ediitorial Reverté, S.A.

Stein, S. (1982). Cálculo y geometría analítica. McGraw-Hill, México. [Tercera edición, traducción del libro Calculus and analytic geometry, 1982].

Stewart, J. (1999). Cálculo, conceptos y contextos. International Thomson, México [Traducción de Calculus concepts and contexts, Brook Cole, 1998].
Swokowski, E. (1982). Calculus with analytic geometry, Second Edition. Prindle, Weber \& Schmidt, USA.

Tall, D., \& Schwarzenberger, R. (1978). Conflicts in the Learning of Real Numbers and Limits. Mathematics Teaching, No. 82, pp. 44-49.

Fecha recibido: Octubre 19 de 2005

Fecha aceptación: Noviembre 25 de 2005 